\title{
Planet Formation in Binary Systems
}

\author{
Wilhelm Kley \\ Computational Physics, Universität Tübingen, Auf der Morgenstelle 10, \\ D-72076 Tübingen, Germany
}

\begin{abstract}
Among the 50 discovered extrasolar planets orbiting mainsequence solar type stars only three are confirmed to be in a binary star system. However, the majority of stars seem to form in binary or even multiple stellar systems. Standard planet formation scenarios consider the creation of planets or planetary systems only for isolated solitary stars. The presence of a companion creates tidal torques on the protoplanetary disk, which may influence the formation process of planets in disks. In this contribution the consequences of the companion's perturbation on the formation scenario of planets is briefly discussed.
\end{abstract}

\section{Planets in Binary Stars}

Among the known 50 extrasolar planets around main sequence stars three (16 Cyg B, 55 Cnc, $\tau$ Boo) are definitely known to be in binary systems with relatively wide orbital separations (see Table 1). Additionally, in one system (Gl 86) there is evidence for a drift in the $\gamma$-velocity (M. Mayor, personal communication) possibly indicating a stellar companion with a distance which may be as close as $10 A U$. If confirmed this very short distance would imply strong consequences for the planetary formation and stability process. It has been argued

Table 1. The properties of known planets in binary stars

\begin{tabular}{cccc|c} 
Parameter & 16Cyg B & 55 Cnc & $\tau$ Boo & Gl 86 \\
\hline Binary $d(\mathrm{AU})$ & 700 & 1150 & 240 & $>10$ \\
M sin $i\left(M_{J}\right)$ & 1.5 & 0.84 & 3.87 & 4 \\
Planet a $(\mathrm{AU})$ & 1.72 & 0.11 & 0.046 & 0.11 \\
Planet e & 0.63 & 0.05 & 0.02 & 0.04 \\
\hline
\end{tabular}

that the high eccentricity $e=0.63$ of $16 \mathrm{Cyg} \mathrm{B}$ may be caused by the presence of the companion star on an inclined orbit through the Kozai mechanism. However, as the ratio of distance $d$ of the secondary over the semi-major axis $a$ of the planets falls into the range

$$
\frac{d}{a}=400-10000
$$


the companion has very little influence on the eccentricity of the planet. The eccentricity of planets can also easily be excited through gravitational interaction of multiple bodies, one of which gets ejected or is scattered into the sun (Weidenschilling \& Marzari, 1996) leaving behind just a single planet. Even though the observations are still sparse the mere existence of extrasolar planets in binary stars demonstrates that formation of planets in multiple systems is not prohibited a priori.

\section{Influence of Companions on Disks}

Planets are believed to have formed within a protoplanetary disk by successive growth processes. Thus, to estimate the influence of a secondary star on the formation process we have to investigate what effect the additional star has on the dynamics of the disk. Let us first consider the case of a disk surrounding one star (circumprimary disk) which is orbited by another, secondary star. The presence of a companion excites tidal waves in the disk which, for a star on a circular orbit, are stationary in a frame corotating with the binary period. This spiral waves carries angular momentum and energy (see eg. Lin \& Papaloizou 1993). As the star orbits the disk beyond the outer disk radius its Keplerian velocity is smaller than that of the disk and the angular momentum carried by the spiral wave is negative respect to the disk material. Dissipation of the wave, for example through shock waves, will slow down the matter in the disk and lead to an inward drift of the material. This in turn truncates the outer edge of the disk.

In case of a binary star within a circumbinary disk, the torques created by the binary lead to angular momentum transfer from the binary's orbit to the disk, and eventually to an inward truncation or clearing of the inner disk (gap formation).

The location of this (inner and outer) truncation radius is determined by the balance of viscous (gap-closing) and gravitational (gap-opening) torques. In linear theory the gravitational potential is expanded into a Fourier series and for each component the effect on the disk is determined. For the general case of a companion on an eccentric orbit this analysis has been performed for circumprimary and circumbinary disks by Artymowicz \& Lubow (1994).

Their main results are displayed in Fig. 1. The label $\mu=0.3$ refers to the reduced mass $\mu=M_{2} /\left(M_{1}+M_{2}\right)$. In case of the circumprimary disk a larger viscosity (lower Reynolds number) leads to a larger truncation radius $r_{t}$ while for a circumbinary disk it reduces the truncation radius. For typical values of protostellar disks $R e \approx 10^{5}$, and for typical binary parameter (see above, $q=0.5, e=0.5)$ we obtain for the circumprimary disk $r_{t} / a=0.17$ and for the circumbinary disk $r_{t} / a=3.0$.

\section{On the Formation of Planets in Tidally Perturbed Disks}

In this final section we consider the restrictions the presence of a secondary companion has on the efficiency of planet formation. The secondary acts as a perturber for the disk and alters directly the environment in which planets form. 

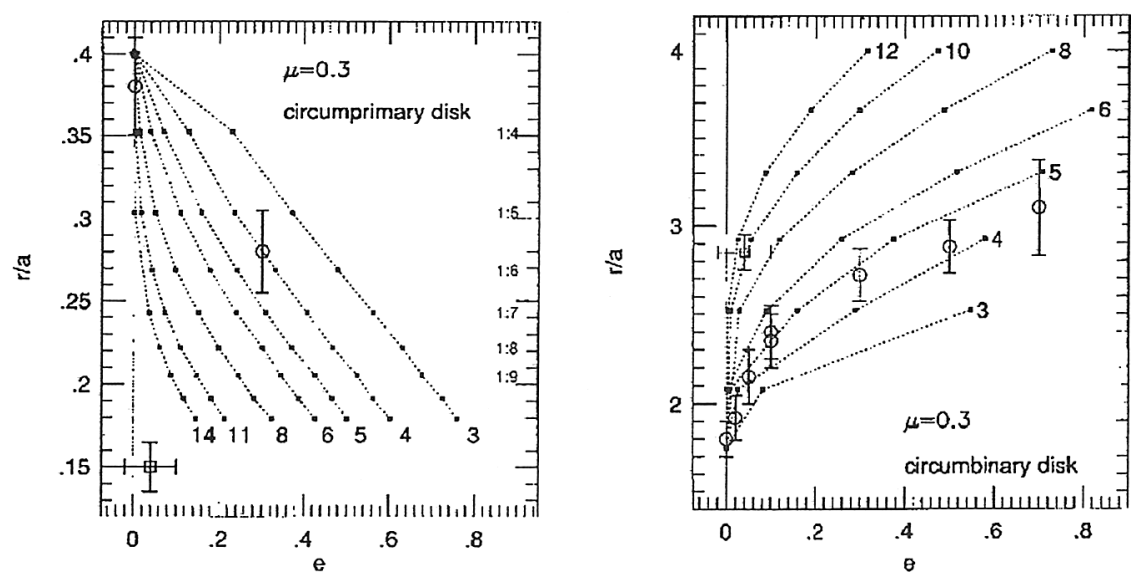

Figure 1. The truncation radius of the disk in units of the semi-major axis of the binary for a mass ratio $q=M_{2} / M_{1}$, and an eccentricity $e=0.5$. Different values of the viscosity are labelled by the logarithm of the Reynolds number in the disk. Adapted from Artymowicz \& Lubow (1994).

\subsection{Planetesimal Disk}

The influence a secondary has on the growth process from planetesimals to planetary embryos is primarily through an increase of the relative motion between particles in a disk. This is caused by an excitation of eccentricity of the particle orbits by the disturbing star. An increased relative velocity yields more destructive collisions and may hinder the growth process considerably. This whole process was studied in detail by Heppenheimer (1978) and Whitmire et al. (1998).

They considered a planetesimal disk at runaway phase where collisions and subsequent merging between different particles lead to a rapid growth. The collisions are only non-destructive if the relative velocities $U_{\text {rel }}$ are smaller than a critical value $U_{c r i t}=100 \mathrm{~m} / \mathrm{s}$. They integrated a 4 -body system consisting of 2 stars and 2 planetesimals for different physical parameters, varying the semi-major axis of the binary stars, their eccentricities, mass ratios, and the initial separation. By analyzing if $U_{r e l}$ was exceeding $U_{c r i t}$, they deduced a critical semi-major axis below which the disturbing companion does not allow for sticking (growing) collisions.

The result implies that for a one-solar mass star the minimum distance to an orbiting companion, such that planetesimals at $1 \mathrm{AU}$ can grow to larger bodies, must always be larger than 16 AU. The critical semi-major axis scales only weakly, $a_{c r i t} \propto(\bar{a} / A U)^{0.8}$, with distance from the star.

\subsection{The Case of L 1551}

Another path of studying the formation of planets in binary stars was taken by A. Nelson (2000), who studied the interaction of two binary stars each of which is surrounded by its own circumstellar disk. The physical motivation is 
based on the radio (VLA) observations of the system L1551 (Rodriguez et al. 1998), which consists of a binary system of two half solar mass stars separated by $50 A U$.

This system is modelled numerically by solving the full hydrodynamic equations for the two disks using the method of Smoothed Particle Hydrodynamics (SPH) using 60,000 particles for each disk, where the continuum equations are modelled by an ensemble of interaction particles.

The model includes the self-gravity of the disks, an ideal equation of state, dissipative heating and radiative cooling.

During the evolution the initially axisymmetric disks (with respect to their central stars) are strongly perturbed at the time of periapse. Spiral waves are induced in them and they are tidally truncated. At times of apoapse the spiral features tend to disappear and the disks become more axisymmetric again. These periodic changes of the distortions alter the internal structure of the disk as well. The question arises what influence do these changes have on the planet formation process. Planet formation is believed to proceed along two different lines: a) Through gravitational collapse of a spiral structure. Instability occurs when the so called Toomre parameter $Q$, which measures the importance of pressure versus gravity, becomes (locally) smaller than unity. Then pressure forces are not sufficient to prevent gravitational collapse. b) Through coagulation of solid material and subsequent gas accretion. As can be inferred from Fig. 2 the $Q$-parameter is always much larger than 1 , indicating a gravitationally stable disk. On the other hand, the temperatures in the disk during periapse are so high that even the most abundant species (water ice) cannot condense to form the seeds for further growth of the planetesimals.

Hence, from the computations one may conclude that, at least for these chosen parameters of the disks, planet formation is inhibited in binary stars. One has to keep in mind however, that the heating and cooling of the disk is treated still approximative and the calculations are only two-dimensional. For larger orbital separations of a few hundred $A U$ say, the effects of the secondary become smaller and planet formation will not be affected.

\subsection{Stability of Orbits}

An important pre-requisite for the formation of planetesimals is the long term stability of their orbits. Dvorak (1986) and Holman \& Wiegert (1999) have studied the restricted 3-body problem, in which the planet is treated as a test particle in the potential of two orbiting stars. They perform numerical integrations and the total integration time covers more than $10^{4}$ orbits of the planet. The mass ratio of the stars, their eccentricity and semi-major axis are varied. The critical distance $a_{c r i t}$ a planet must have to be on a stable orbit is determined as a function of these orbital parameters of the binary star.

Two different configurations of orbits have been studied. Following the designation of Dvorak (1986), the first are planetary or P-type orbits which are well outside the binary. The second type studied are S-type orbits, where the planet orbits near one of the stars, with the second star to be considered as a perturber. For P-type orbits there exist a minimum $a_{\text {crit }}$ a planet must have to be on a stable orbit around the binary. For S-type orbits there is a maximum $a_{\text {crit }}$ such that the perturbations of the perturber remain finite. The S-type 


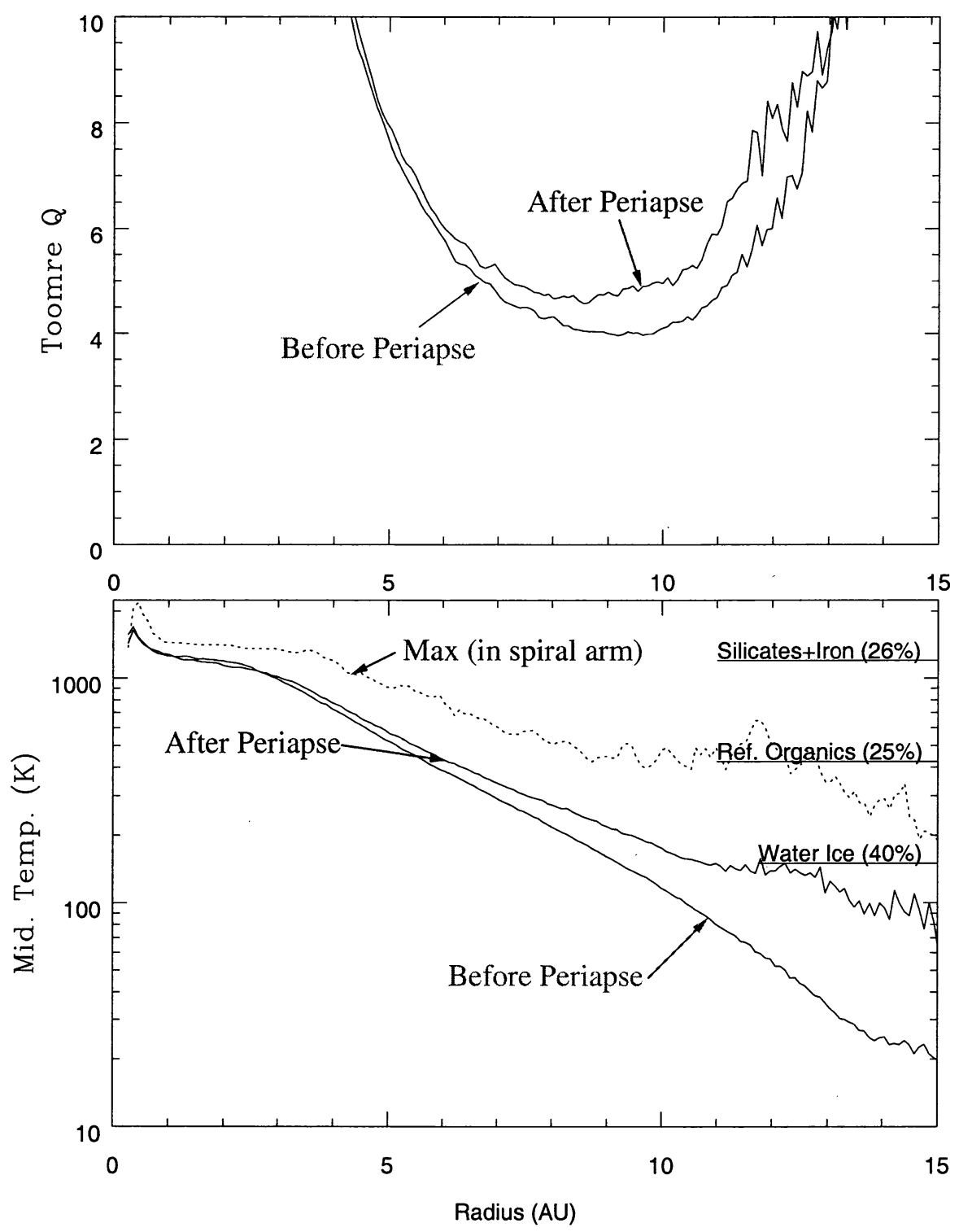

Figure 2. Azimuthally averaged Toomre-parameter $Q$ (top) and the midplane temperature in the disk versus radius. Indicated are the vaporization temperatures of various species. 
orbits are certainly more relevant to the properties of the observed extrasolar planets (see table 1).

For an average eccentricity of $e=0.5$ one finds for S-type orbits $a_{\text {crit }} \approx 0.14$. All observed extrasolar planets have semi-major axis well below this limit. For P-type orbits the minimum distance a planet must have from the binary is $a_{c r i t} \approx 3.6$. This value lies beyond the inner truncation radius of a circumbinary disk $\left(r_{\text {gap }}=3.0\right)$.

\subsection{The Evolution of an Embedded Planet in a Binary Star}

Another approach to analyze the influence of a companion on the formation of a massive planet has been taken by Kley $(2000 \mathrm{~b})$ who studied the evolution of a massive planet still embedded in a protoplanetary disk.

A planet with the mass $1 M_{J u p}$ is placed initially on a circular orbit around a $1 M_{\odot}$ star at a distance of $a_{J}=5.2 \mathrm{AU}$. The surrounding protostellar disk has a mass of $M_{d}=0.01 M_{\odot}$ within $1-20 A U$, a surface density profile $\Sigma(r) \propto r^{-1 / 2}$, and a Reynolds number of $10^{5}$. The secondary has a given fixed mass of $M_{2}=$ $0.5 M_{\odot}$ and an eccentricity of $e_{2}=0.5$. The semi-major axis $a_{2}$ of the secondary is varied from about 50 to $100 A U$ for different models. To minimize initial disturbances, the secondary is placed at apastron in the beginning. During the evolution, the planet may accrete material from the disk and increase its mass. These evolution calculation are similar to the migration computations as presented by Kley $(1999,2000 \mathrm{a})$ and Nelson et al. (2000).

In the presence of the secondary the disk becomes truncated at the outer radius. At the same time the planet will truncate the disk from the inner side such that the disk material will be confined to a narrow ring (see Fig. 3). The obtained values for the outer truncation radius agree favourably with the value $r_{r} / a=0.17$ as obtained above. The very steep density and pressure gradient near the planet lead to an increased mass accretion rate onto the planet, and to a faster inward migration, cf. Fig. 3.

The reduced migration time may then also reduce the growth of a protoplanet and planet formation will again be inhibited by the presence of a companion.

\subsection{Planet Formation in Circumbinary Disks}

In circumbinary disks the angular momentum barrier causes material to pile up near the inner edge of the gap, although there may be some accretion onto the binary star taking place (see contribution by Mathieu and by Stassun, this volume). This accumulation of material may be favourable for planet formation mainly for two reasons: a) The possibility of gravitational instability and collapse is increased. This scenario has been studied by Laughlin and Lin and preliminary results including the self-gravity of the disk indicate a Toomre $Q$-value of around 1. For rapidly cooling isothermal disks fragments can form which may be tossed out as blobs to possibly form free-floating objects of very low mass; and b) As the flow is super-Keplerian near the inner edge of the disk pressure effects will cause a particle accumulation near the inner edge of the disk, which could increase planet formation. However, the perturbations by the star will also stir up the particles increasing their relative velocities (see above). So, the net effect on the efficiency of planet formation is not clear. 

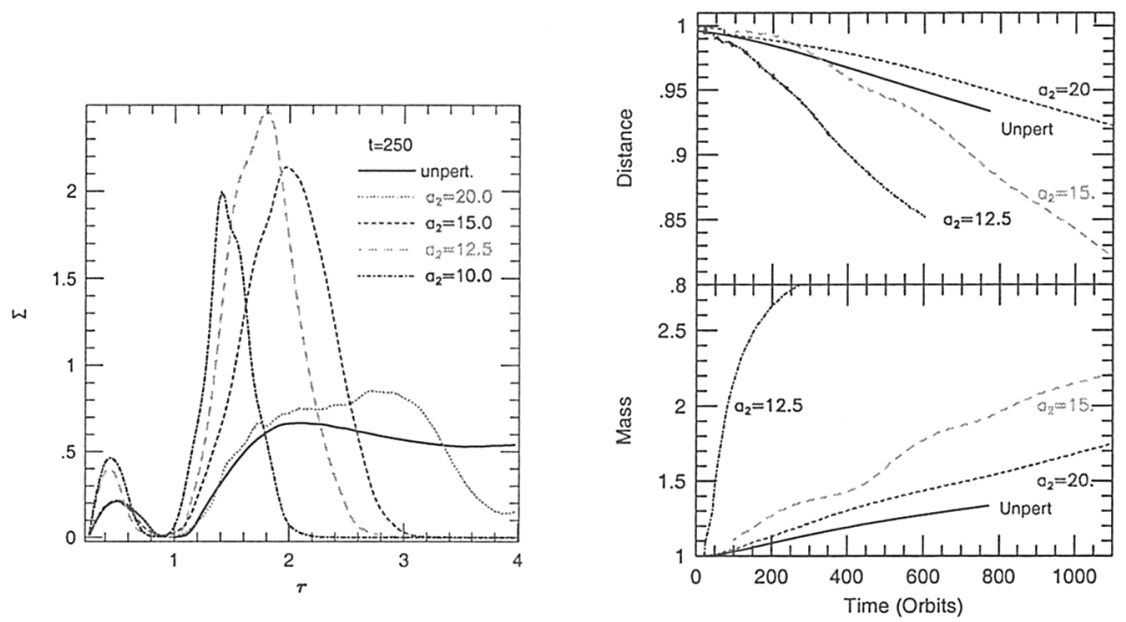

Figure 3. Left: Azimuthally averaged surface density for models with varying distance $\left(a_{2}\right)$ of the secondary, given in units of the planetary distance $(5.2 A U)$, after 250 orbits of the planet. The solid black line indicates the initial surface density profile. Right: Evolution of the mass (bottom) and semi-major axis (top) of the planet. The evolution of an unperturbed planet (i.e. no secondary star) which is described in detail in Nelson et al. (2000) is given by the solid lines (denoted "Unpert").

\section{Summary and Outlook}

We have seen that the predominant effect a companion has on the efficiency of planet formation is a negative one. The stirring induced by the secondary star heats up the disk, making it more difficult for particles to stick together and at the same time inhibiting gravitational collapse. However, since planet formation in itself is still a poorly understood process new detailed numerical computations are needed to arrive at a more detailed understanding of the process. Some of the problems addressed may include: i) a detailed calculation of the torques acting on the planet to estimate timescales of migration and the influence of the secondary, ii) more elaborate thermodynamics in disks (shock, viscous dissipation, cooling, magnetic fields) to calculate the efficiency of fragmentation and collapse, iii) multiple planet calculations (in binary systems) to study the evolution of the semi-major axis, the eccentricity, and the efficiency of ejections. To achieve these goals multi-dimensional (MRHD, magneto-radiation-hydro) computations will be required.

\section{References}

Artymowicz, P., \& Lubow, S. H. 1994, ApJ, 421, 651

Dvorak, R. 1986, A\&A, 167, 379 
Heppenheimer, T. A. 1978, A\&A, 65, 421

Holman, M. J., \& Wiegert, P. A. 1999, AJ, 117, 621

Kley, W. 1999, MNRAS, 303, 696

Kley, W. 2000a, 313, L47

Kley, W. 2000b, in preparation

Lin, D. N. C., \& Papaloizou, J. C. B. 1993, in Protostars and Planets III, ed. E. H. Levy \& J. I. Lunine, (Tucson: Univ. Arizona Press), 749

Nelson, A. 2000, ApJ, 537, L65

Nelson, R. P., Papaloizou, J. C. B., Masset, F., \& Kley, W. 2000, MNRAS, 318, 18

Rodriguez, L. F., D'Alessio, P., Wilner, D. J., Ho, P. T. P., Torrelles, J. M., Curiel, S., Gomez, Y., Lizano, S., Pedlar, A., Canto, J., \& Raga, A. C. 1998, Nature, 395, 355

Weidenschilling, S. J., \& Marzari, F. 1996, Nature, 384, 619

Whitmire, D. P., Matese, J. J., Criswell, L., \& Mikkola, S. 1998, Icarus, 132, 196

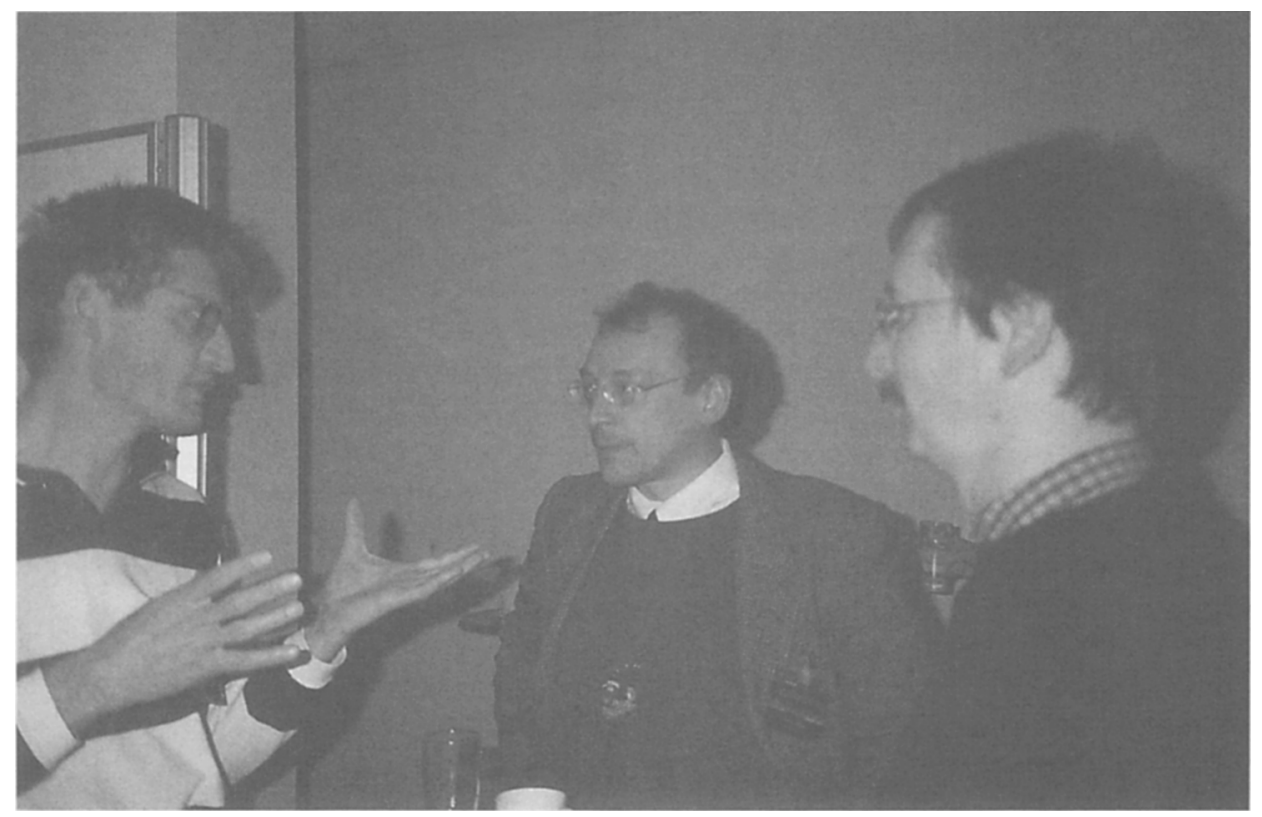

Wilhelm Kley, Tomek Plewa and Thomas Henning 